\title{
IDOSOS VINCULADOS À ATENÇÃO DOMICILIAR: CARACTERIZAÇÃO SOCIODEMOGRÁFICA
}

\section{Êmilly Barcelos Petter ${ }^{1}$; Claudia Maria Ferrony Rivas ${ }^{2}$; Eduardo Marques Machado2; Natalia Cassol Bolzan2; Clandio Timm Marques3; Naiana Oliveira dos Santos ${ }^{4}$}

\section{RESUMO}

Objetivo: analisar e discutir a caracterização sociodemográfica de idosos na Atenção Domiciliar da Atenção Primária à Saúde. Metodologia: estudo transversal de caráter descritivo. A coleta de dados foi realizada via contato telefônico com idosos vinculados à Atenção Domiciliar tipo 1 e foi utilizado um questionário sociodemográfico. Para realizar a análise dos dados foi utilizada a estatística descritiva. Resultados: foram avaliados 108 idosos, com média de idade de 78,7 anos e desvio padrão de 9,3 anos, destes, a maioria era do sexo feminino $(71,3 \%)$. A maior parte da amostra relatou ser casado $(44,4 \%)$, não ser alfabetizado $(65,7 \%)$ e possuir entre 1-2 salários mínimos $(54,6 \%)$. Observou-se alta prevalência de comorbidades, sendo as principais Hipertensão Arterial Sistêmica, Diabetes Mellitus, Artrite/artrose e Depressão. Conclusão: torna-se visível a importância do rastreamento das variáveis sociodemográficas e condições de saúde dos idosos da Atenção Domicilar, pois auxilia no planejamento em saúde e desenvolvimento de ações específicas voltadas à este grupo etário.

Palavras-chave: Atendimento Domiciliar; Atenção Primária à Saúde; Enfermagem; Saúde do Idosos.

Eixo Temático: Atenção Integral e Promoção à Saúde.

\footnotetext{
${ }^{1}$ Acadêmica do Curso de Enfermagem da Universidade Franciscana - UFN. E-mail: emilly.bpetter@ufn.edu.br

${ }^{2}$ Acadêmicos do Curso de Enfermagem da Universidade Franciscana - UFN. E-mail: natalia cassol@ufn.edu.br; claudiamfrivas@gmail.com; eduardomarques051@gmail.com

${ }^{3}$ Docente do Curso de Matemática da Universidade Franciscana - UFN. E-mail: clandiomarques@gmail.com

${ }^{4}$ Orientadora. Doutora em Enfermagem. Professora do Curso de Enfermagem da Universidade Franciscana - UFN. E-mail: naiana.oliveira@ufn.edu.br
} 


\section{INTRODUÇÃO}

É notável as transformações nos padrões demográficos, sociais, tecnológicos, climáticos e, principalmente, na estrutura etária da população. No Relatório Mundial de Envelhecimento e Saúde da Organização Mundial da Saúde (OMS) (2015), é exposto diferentes assuntos relacionados a essas mudanças no contexto do processo do envelhecimento, além de preconizar a necessidade de reformulação e alterações nas políticas em saúde e os serviços de saúde para essa população.

Há anseio em novas adaptações no sistema de saúde para garantir melhores qualidades no acesso e utilização desses serviços. Proporcionar ao idoso não somente viver por mais tempo, mas também, de forma ativa e saudável por meio da atenção integral, cuidado do seu bem-estar, qualidade de vida, manutenção funcional e prevenção às condições crônicas em saúde (OMS, 2015; MIRANDA, 2016).

O idoso consome mais serviços de saúde, as internações hospitalares são mais frequentes e o tempo de ocupação do leito é maior quando comparado a outras faixas etárias. Esse fato é decorrência do padrão das doenças dos idosos, que são crônicas e múltiplas, e exigem acompanhamento constante, cuidados permanentes, medicação contínua e exames periódicos (VERAS; OLIVEIRA, 2016).

Nesse sentido, a Atenção Domiciliar (AD) vem como uma oportunidade de agregar a atenção completa e contínua às ações de saúde no contexto do usuário, no seu próprio domicílio. As visitas domiciliares mostram-se como uma ferramenta de cuidado integral, uma técnica de intervenção em saúde bem como algo que aproxima a família do serviço de saúde e do profissional de referência para o cuidado de quem se encontra vulnerável (ROCHA et al., 2017).

A AD promove o vínculo entre o profissional de saúde e o idoso, em principal, por meio dessas visitas, e deste modo, desenvolver a aproximação da realidade de vida do idoso com a coordenação da otimização do cuidado. Na compreensão da singularidade desta fase da vida, pode-se dizer que o ato de visitar a residência de um usuário e estabelecer um processo de cuidado respeitando suas necessidades, é algo que situa a $A D$ como uma ferramenta que extrapola a dimensão técnica do 
cuidado e mostra-se como uma ferramenta, também, humana (MARQUES et al. 2020).

Deste modo, o presente trabalho tem como objetivo analisar e discutir a caracterização sociodemográfica de idosos vinculados à atenção domiciliar da Atenção Primária à Saúde.

\section{METODOLOGIA}

Trata-se de um estudo transversal com abordagem quantitativa e caráter descritivo, o qual foi realizado via contato telefônico com idosos vinculados à $A D$ da Atenção Primária à Saúde (APS) do município de Santa Maria/RS. As pesquisas transversais indicam a frequência com que determinada doença ou outro efeito de interesse, ocorrem em uma determinada população ou área geográfica num determinado momento. São feitas análises comparativas entre as variáveis ecológicas, geralmente por meio de correlação entre indicadores de condição de vida e indicadores de saúde (FREIRE; PATTUSSI, 2018).

Os dados deste estudo são oriundos de um estudo maior intitulado "Funcionalidade global e os sistemas funcionais de idosos vinculados à atenção domiciliar da atenção básica". A coleta de dados foi realizada com idosos cadastrados na AD tipo 1 de quatro Estratégias de Saúde da Família (ESFs), as quais possuíam mais idosos cadastrados. Incluiu-se idosos com idade igual ou superior a 60 anos, de ambos os sexos e que estavam cadastrados na AD1 das referidas ESFs. Foram excluídos aqueles que não foi possível realizar o contato telefônico após três tentativas em períodos e horários diferentes, idosos institucionalizados ou hospitalizados no momento e idosos que possuíam incapacidade ou insuficiência cognitiva e com diagnóstico de demência avançada.

Havia 247 usuários cadastrados na AD1 nas ESFs informadas, porém somente 108 indivíduos se enquadraram nos critérios de inclusão e participaram do estudo, sendo que 139 idosos foram excluídos conforme os critérios de exclusão. A coleta de dados foi realizada por meio de uma entrevista estruturada via contato telefônico no período de julho a agosto de 2021. Utilizou-se um questionário sociodemográfico desenvolvido pelos pesquisadores com as seguintes variáveis: 
idade, sexo, estado civil, nível de escolaridade, renda familiar, comorbidades e o tempo de acompanhamento na AD.

Durante a aplicação do questionário o cuidador poderia auxiliar o pesquisador nos questionamentos aos idosos. Nas entrevistas estavam presentes pelo menos um pesquisador, o qual realizou o contato telefônico, e o idoso juntamente com seu cuidador ou familiar para auxiliar nas respostas. Os termos de consentimento foram gravados pelo microfone de um computador, através do aplicativo "Telegram", o qual se baseia em mensagens instantâneas com armazenamento em nuvem. Para realizar a análise dos dados foi utilizada a estatística descritiva.

O projeto foi submetido e aprovado pelo Comitê de Ética em Pesquisa da Universidade Franciscana, sob o número 3.308.174. Os participantes tiveram clareza dos objetivos da pesquisa, por meio da leitura do Termo de Consentimento Livre e Esclarecido (TCLE) pelo pesquisador, conforme resolução do Conselho Nacional de Saúde 466/12. Destaca-se que os nomes dos participantes foram mantidos anônimos para preservar suas identidades. No texto, os idosos foram identificados por U1, U2 e assim sucessivamente.

\section{RESULTADOS E DISCUSSÕES}

Participaram deste estudo 108 idosos domiciliados. A média de idade da população estudada foi de 78,7 anos com desvio padrão de 9,3 anos. A menor idade encontrada foi de 60 anos, e a maior de 100 anos. A partir disso, é possível observar a diferença de idades entre os idosos coletados, onde desde a idade máxima necessita de atendimento domiciliar quanto a idade mínima, ressaltando que são considerados idosos aqueles acima de 60 anos.

O acelerado envelhecimento da população brasileira juntamente com 0 aumento da longevidade, traz consigo diversas consequências na estruturação das Redes de Atenção à Saúde (RAS), isso devido ao crescimento das incapacidades funcionais e doenças crônicas. Dessa forma, há diversas demandas e um maior uso dos serviços de saúde, principalmente da Atenção Básica $(A B)$, onde é necessário realizar o atendimento domiciliar aos idosos (MORAES et al., 2018). 
Dos idosos participantes, $28,7 \%$ são do sexo masculino, enquanto a prevalência da população do estudo foi do sexo feminino, com total de $71,3 \%$. Isso pode-se justificar devido a população feminina procurar mais os serviços de saúde que a população masculina.

Em um estudo, Levorato et al. (2014), também aponta que as mulheres buscaram 1,9 a mais os serviços de saúde do que os homens. Ademais, outras pesquisas (RIBEIRO, 2005; BRASIL, 2008; COUTO, 2010; CARNEIRO et al., 2019) ressaltam que os homens têm dificuldade de procurar os serviços da AP, vindo ao encontro do impasse do autocuidado e consequentemente, suscetíveis a doenças graves e crônicas.

A baixa procura dos homens pelos serviços de saúde é influenciada por um modelo hegemônico de masculinidade, no qual prevalecem as noções de virilidade, força e invulnerabilidade (GOMES, 2007). Dentre outros motivos que levam o sexo masculino a essa resistência, há os desafios que abrange a APS desde a ampliação do acesso e abertura, profissionais qualificados para a promoção, prevenção e tratamento específico, desde maiores ações favoráveis para essa população (CARNEIRO et al., 2019).

Quanto ao estado civil dos idosos participantes, apenas $4,6 \%$ eram solteiros, $7,5 \%$ divorciados ou separados, viúvo $43,5 \%$ e o maior percentual foi de idosos casados, com 44,4\%. Os idosos com companheiro apresentam melhor bem-estar físico e psicológico devido ao maior apoio social e familiar, possuindo menor probabilidade de relatar redes sociais de baixa qualidade (FHON et al., 2018)

A partir da análise do nível de escolaridade dos idosos deste estudo, foi possível perceber que a maior parte da população estudada não é alfabetizada, $65,7 \%$ com fundamental incompleto. Ensino fundamental completo $21,3 \%$ e com médio incompleto e médio completo contabilizou 6,5\%.

Cabe destacar, que o nível de escolaridade está intimamente relacionado a uma melhor qualidade de vida, uma vez que proporciona ao indivíduo maior autonomia e independência de atividades, principalmente em idosos, visto que o conhecimento a respeito das condições da própria saúde proporciona o 
desenvolvimento do empoderamento do sujeito e, repercute diretamente no aumento da expectativa de vida desta população (LINARD et al., 2021).

A renda familiar total da maioria dos idosos foi de 1-2 salários mínimos (54,6\%), seguido de 2-3 salários mínimos (31,1\%), maior que 3 salários mínimos $(10,7 \%)$ e com baixa prevalência daqueles com menos que 1 salário mínimo (3,9\%). Pode-se explicar que a maioria apresenta renda intermediária pois reside com familiares que contribuem na renda domiciliar. Ressalta-se que nesta questão, cinco usuários não tiveram o interesse em relatar a renda familiar.

O faturamento familiar representa um fator determinante na situação de saúde do idoso, possivelmente nesta fase da vida exista uma necessidade maior de medicamentos, presença de doenças crônicas, alimentação diferenciada e outros custos que o processo de limitação física acarreta. Além disso, devido às diversas mudanças ocorridas nos arranjos familiares nos últimos tempos, o idoso pode se deparar com uma realidade na qual se vê obrigado a amparar familiares desempregados ou doentes (SANTOS et., 2018).

Apresentou-se alta prevalência de idosos com comorbidades, onde 98,1\% possuíam. Em relação às comorbidades apresentadas pelos idosos, 63,9\% relataram possuir Hipertensão Arterial Sistêmica (HAS). Um estudo realizado pela Pesquisa Nacional de Saúde, apontou uma maior prevalência de HAS com idosos de 75 anos ou mais (55,5\%), seguido dos com 65 a 74 anos $(52,7 \%)$ e com 60 a 64 anos (44,4\%) (IBGE, 2013).

Quanto a Diabetes Mellitus (DM), 38\% confirmaram que possuíam. O DM em idosos está relacionado a um risco maior de morte prematura, maior associação com outras comorbidades e com as grandes síndromes geriátricas, além dos prejuízos em relação à capacidade funcional, autonomia e qualidade de vida (RAMOS et al., 2017).

A presença de condições crônicas (HAS e DM) aumentou em cerca de $40 \%$ a probabilidade de recebimento de atenção domiciliar entre os idosos (WACHS et al., 2016). Outros estudos (PICCINI et al., 2006; THUMÉ et al., 2010) também ressaltam e justificam que por haver uma maior necessidade de saúde, resulta na expressiva utilização dos serviços de saúde decorrentes das patologias crônicas. 
Outra comorbidade apresentada no estudo foi artrite e/ou artrose, onde 32,4\% possuíam. Fechine e Trompieri (2012), apontam que com o envelhecimento ocorrem algumas alterações referentes a articulação em virtude do lento reparo tecidual, diminuição no número de vasos sanguíneos, rigidez da cartilagem articular, redução na produção de líquido sinovial e redução da amplitude de movimento das articulações devido à menor flexibilidade dos ligamentos e tendões.

Além disso, as alterações do sistema muscular, como a fraqueza dos músculos em consequência da redução da força muscular, contribuem para a menor movimentação das articulações (PINHEIRO et al., 2019). Nesse sentido, Volpon (2014) destaca que as doenças articulares, como a artrite, caracterizada pela inflamação das articulações, acomete muito nos indivíduos ao envelhecer. Associada ao excesso de peso corporal, trabalhos repetitivos, idade avançada, lesões e dentre outros, pode-se destacar alguns tipos de artrite como a reumatóide, artrite gotosa (gota), osteoartrite (artrose) e mais.

Para Drummond e Alves (2012) essas doenças crônicas degenerativas estão relacionadas ao envelhecimento e causam limitação funcional, em que, consequentemente, as incapacidades funcionais aumentam o grau de dependência do idoso. Os fatores extrínsecos como demográfico, cultural e socioeconômico também podem interferir na funcionalidade da pessoa idosa, observando a alimentação, fumo, ingestão de bebida alcoólica, pontos do estilo de vida que estão inseridos nesse contexto.

Quanto à depressão houve $19,4 \%$ de idosos com o transtorno. Alguns participantes relatam que desenvolveram a depressão pela perda de algum ente querido ou devido a pandemia, por não terem contato com amigos e familiares. A população idosa apresenta maior vulnerabilidade para problemas de saúde, em especial, no que diz respeito aos transtornos de humor, nesta fase os indivíduos podem ter sentimentos negativos sobre o envelhecimento, achar que não são mais úteis à sociedade, aos seus entes queridos, sentir que a vida está se findando, alguns chegam a se isolar, não ter apreço pelo convívio social. Para mais, costumam ser acometidos por doenças decorrentes da idade. Sentimentos assim 
podem desencadear grande tristeza podendo resultar em um quadro depressivo (SANTOS et al., 2009).

O tempo de acompanhamento dos idosos na AD1 teve um tempo máximo de 17 anos e mínimo de 1 mês. Apenas 11 usuários não souberam relatar o tempo exato que estão em acompanhamento domiciliar.

\section{CONCLUSÃO}

O presente estudo visou analisar as variáveis sociodemográficas e condições de saúde de 108 idosos vinculados a AD1 do município de Santa Maria/RS. Os resultados encontrados demonstram a prevalência significativa do sexo feminino, estado civil casado, analfabetismo, renda domiciliar média entre 1-2 salários mínimos e elevado índice de patologias. A comorbidade de maior prevalência foi a HAS, seguido por DM, artrite/artrose e por última depressão.

Com isso, foi possível perceber que a maioria dos idosos faziam parte da AD1 por possuírem comorbidades que requerem cuidados de menor complexidade e frequência, as quais se caracterizam como doenças crônicas não transmissíveis (DCNT). Assim, destaca-se a importância do rastreamento das variáveis sociodemográficas e condições de saúde dos idosos da $A D$, pois auxilia no planejamento em saúde e desenvolvimento de ações específicas voltadas a este grupo etário que se encontra em constante crescimento.

Como limitação na realização desta pesquisa, destaca-se que por ter sido realizada via contato telefônico alguns participantes ficaram receosos de informar a renda domiciliar, e desta forma, sugere-se que novos estudos realizados de maneira remota evitem utilizar tal variável.

\section{AGRADECIMENTOS}

Agradeço ao Programa Institucional de Bolsas de Iniciação Científica (PROBIC) da Universidade Franciscana (UFN) pela oportunidade e suporte na realização e desenvolvimento do experimento e na análise dos dados.

\section{REFERÊNCIAS}

BRASIL, Secretaria de Atenção à Saúde, Departamento de Ações Programáticas Estratégicas. Política Nacional de Atenção Integral à Saúde do Homem 
(Princípios e Diretrizes) [Internet]. Brasília: Ministério da Saúde; 2008. Disponível em:

https://bvsms.saude.gov.br/bvs/publicacoes/politica nacional atencao homem.pdf Acesso em: 23 set. 2021

CARNEIRO, V. S. M.; ADJUTO, R. N. P.; ALVES, K. A. P. Saúde do homem: identificação e análise dos fatores relacionados à procura, ou não, dos serviços de atenção primária. Arq. Cienc. Saúde UNIPAR, Umuarama, v. 23, n. 1, p, 35-40, 2019.

em:

https://www.revistas.unipar.br/index.php/saude/article/view/6521/3728

Acesso em: 19 set. 2021

COUTO, M. T. et al. O homem na atenção primária à saúde: discutindo (in)visibilidade a partir da perspectiva de gênero. Interface - Comunicação, Saúde, Educação, v. 14, n. 33, p. 257-70, 2010. Disponível em: https://www.scielo.br/j/icse/a/5Dgnp7BfTBDtcfkz4KMMxsC/abstract/?lang=pt

Acesso em: 23 set. 2021

DRUMMOND, A.; ALVES, E. D. Determinantes na funcionalidade de idosos da estratégia saúde da família - revisão integrativa. Tempus - Actas de Saúde Coletiva, v. 6, n. 4, p. 113-22, 2012. Disponível em: https://www.tempusactas.unb.br/index.php/tempus/article/view/1209/1094

Acesso em: 23 set. 2021

FHON, J. R.S. et al. Fatores associados à fragilidade em idosos: estudo longitudinal. Rev saude publica (Online), 2018. Disponível em: https://www.scielo.br/j/rsp/a/bmbq45Lb6fZvgyHSRSZyXFd/?format=pdf\&lang=pt Acesso em: 23 set. 2021

FECHINE, B. R. A; TROMPIERI, N. O processo de envelhecimento: as principais alterações que acontecem com o idoso com o passar dos anos. Rev. Cien. Int., v. 1, n. $7,2012 . \quad$ p. $2012-194, \quad$ Disponível em: http://www.fonovim.com.br/arquivos/534ca4b0b3855f1a4003d09b77ee4138Modifica----es-fisiol--gicas-normais-no-sistema-nervoso-do-idoso.pdf Acesso em: 19 set. 2021

FREIRE, M.C.M.; PATTUSSI, M.P. Tipos de estudos. IN: ESTRELA, C. Metodologia científica. Ciência, ensino e pesquisa. $3^{\underline{a}}$ ed. Porto Alegre: Artes Médicas, p.109-127, 2018.

GOMES, R.; NASCIMENTO, E.F.; ARAÚJO, F. C. Por que os homens buscam menos os serviços de saúde do que as mulheres? As explicações de homens com baixa escolaridade e homens com ensino superior. Cad Saúde Publica, v.23, n. 3, p.565-74, $2017 . \quad$ Disponível em: https://www.scielo.br/j/csp/a/rQC6QzHKh9RCH5C7zLWNMvJ/abstract/?lang=pt Acesso em: 19 set. 2021 
IBGE. Instituto Brasileiro de Geografia e Estatística. Pesquisa Nacional de Saúde 2013. Percepção do Estado de Saúde, Estilos de Vida e Doenças Crônicas. Rio de Janeiro, 2013.

LEVORATO, C. D. et al. Fatores associados à procura por serviços de saúde numa perspectiva relacional de gênero. Ciência \& Saúde Coletiva [online]. v. 19, n. 4, pp. 1263-1274, $2014 . \quad$ Disponível em: https://www.scielo.br///csc/a/8cp6H8fy9rSpQvGG3WcYXKB/?lang=pt Acesso em: 19 set. 2021

LINARD, L.L.P.; FREITAS, F.F.Q.; BELEZA, C.M.F; FERNANDES, M. C.; SOARES, S.M. Caracterização do perfil epidemiológico de idosos cadastrados na atenção primária à saúde. R. pesq.: cuid. fundam. (online) v. 13, p. 524-30, 2021. Disponível em: http://seer.unirio.br/cuidadofundamental/article/view/9280/pdf 1 Acesso em: 23 set. 2021

MARQUES, F. P.; BULGARELLI, A. F. Os sentidos da atenção domiciliar no cuidado ao idosos na finitude: a perspectiva humana do profissional do SUS. Ciência \& Saúde Coletiva, v. 26, n.6, p. 2063-72, 2020. Disponível em: https://www.scielo.br///csc/a/3K7JYrSQmmc79t7nvR5C8YS/?lang=pt

Acesso em: 14 set. 2021

MIRANDA, G. M. D.; MENDES, A. C. G.; SILVA, A. L. A. da. O envelhecimento populacional brasileiro: desafios e consequências sociais atuais e futuras. Rev. Bras. Geriatr. Gerontol., Rio de Janeiro, v.19, n.3, p. 507-519, 2016. Disponível em: https://www.scielo.br/j/rbgg/a/MT7nmJPPRt9W8vndq8dpzDP/abstract/?lang=pt Acesso em: 14 set. 2021

MORAES, E. N.; AZEVEDO, R. S.; MORAES, F. L.; PEREIRA, A. M. V. B. Secretaria de Estado da Saúde do Paraná. Superintendência de Atenção à Saúde. Avaliação multidimensional do idoso. SAS. - Curitiba: SESA, 113p., 2018. Disponível em: https://www.saude.pr.gov.br/sites/default/arquivos restritos/files/documento/202004/avaliacaomultiddoidoso 2018 atualiz.pdf Acesso em: 14 set. 2021

OMS. Organização Mundial da Saúde. Relatório mundial de envelhecimento e saúde. Genebra: OMS; 2015.. Disponível em: https://sbgg.org.br/wpcontent/uploads/2015/10/OMS-ENVELHECIMENTO-2015-port.pdf

Acesso em: 14 set. 2021

PICCINI, R. X. et al. Necessidades de saúde comuns aos idosos: efetividade na oferta e utilização em atenção básica à saúde. Ciênc Saúde Coletiva, v.11, n.3, p. $657-67$, 2006. em: https://www.nescon.medicina.ufmg.br/biblioteca/imagem/0413.pdf Acesso em: 19 set. 2021 
PINHEIRO, S. C. B.; BARRENA, H. C.; MACEDO, A. B. Alterações articulares causadas pelo envelhecimento e seus impactos para a autonomia do idoso. Arquivos do MUDI, v. 23, n. 3, p. 35-45, 2019. Disponível em: https://periodicos.uem.br/ojs/index.php/ArgMudi/article/view/51510

Acesso em: 22 set. 2021

RAMOS, R. S. P. S. et al. Factors associated with diabetes among the elderly receiving care at a specialized gerontology-geriatric outpatient clinic. Revista Brasileira de Geriatria e Gerontologia [online], v. 20, n. 3, p. 363-73, 2017. Disponível em: https://www.scielo.br/j/rbgg/a/NRTBYLzwxdSKfbb5vsMvHFN/?lang=pt Acesso em: 19 set. 2021

RIBEIRO, M. M. Utilização de Serviços de Saúde no Brasil: uma investigação do padrão etário por sexo e cobertura por plano de saúde [dissertação]. Belo Horizonte: Universidade Federal de Minas Gerais; 2005.

ROCHA, K.B., CONZ, J.; BARCINSKI, M.; PAIVA, D.; PIZZINATO, A. A visita domiciliar no contexto da saúde: uma revisão de literatura. Psicologia, Saúde \& Doenças, v. $18, \quad$ n. 1 , p. 170-185, 2017. Disponível em: https://www.redalyc.org/pdf/362/36250481015.pdf Acesso em: 14 set. 2021

SANTOS, F.H. et al. Envelhecimento: um processo multifatorial. Psicologia em Estudo, v. 14, m. 1, p. 3-10, 2009. Disponível em: https://www.scielo.br/j/pe/a/FmvzytBwzYqPBv6x6sMzXFq/abstract/?lang=pt Acesso em: 22 set. 2021

SANTOS, G. S.; CUNHA, I. C. K. O. Prevalência e fatores associados à hipertensão em idosos de um serviço de atenção primária. Rev. Família, Ciclos de Vida e Saúde no Contexto Social, vol. 1, 2018. Disponível em: https://www.redalyc.org/articulo.oa?id=497955551007 Acesso em: 16 set. 2021

THUMÉ, E.; FACCHINI, L. A.; TOMASI, E.; VIEIRA, L. A. S. Assistência domiciliar a idosos: fatores associados, características do acesso e do cuidado. Rev Saúde Pública, v. 44, n. 6 , p. 1102-11, 2010. Disponível em: https://www.scielo.br/j/rsp/a/6J35tXqqM9WpnPsSxXPP6Sn/?lang=pt

Acesso: 23 set. 2021

VERAS, R.P.; OLIVEIRA, M.R. Linha de cuidado para o idoso: detalhando o modelo. Rev. bras. geriatr. gerontol., Rio de Janeiro, v. 19, n.6, p. 887-905, 2016. Disponível em: https://www.scielo.br/j/rbgg/a/P4THmK5H3nzDZby7fr6ssWk/?lang=pt Acesso em: 14 set. 2021

VOLPON, J. B. Fundamentos de ortopedia e traumatologia. São Paulo: Atheneu, p. 432, 2014. 


\section{QUFN}

WACHS, L. S.; NUNES, B. P.; SOARES, M. U. S.; FACCHINI, L. A.; THUMÉ, E. Prevalência da assistência domiciliar prestada à população idosa brasileira e fatores associados. Cad. Saúde Pública, Rio de Janeiro, v. 32, n. 3, 2016 Disponível em: https://www.scielo.br/i/csp/a/BRMgtbjG85DmCg3ZDzW99GD/?lang=pt Acesso em: 23 set. 2021 